\title{
Phase space lattices with threefold symmetry
}

\author{
Michael Wilkinson $†$ and Elizabeth J Austin $\dagger$ \\ Institute for Theoretical Physics, University of California, Santa Barbara, CA 93106, USA
}

Received 16 November 1989

\begin{abstract}
This paper presents a semiclassical analysis of the spectrum of the Hamiltonian

$$
\hat{\mathrm{H}}=\cos (\hat{x}-\theta)+\cos \frac{1}{2}(\hat{x}+\sqrt{3} \hat{p})+\cos \frac{1}{2}(\hat{x}-\sqrt{3} \hat{p})
$$

which is a model for Bloch electrons in a magnetic field. The energy levels are determined, to a first approximation, by Bohr-Sommerfeld quantisation. Because of the translational symmetry in phase space, this leads to a lattice of infinitely degenerate states. This degeneracy is lifted by tunnelling effects, which can be described by an effective Hamiltonian of the same form as that above, but with different values of $\theta$ and $\hbar$. The calculation of the effective Hamiltonian therefore defines a renormalisation-group transformation, and it predicts that the spectrum has a complex recursive structure, which is confirmed by numerical experiments.

The results complement earlier work on lattices with fourfold symmetry, in which a similar type of spectrum occurs: the greater complexity of the problem in lattices with threefold symmetry necessitated developing simpler, canonically invariant, methods of analysis.
\end{abstract}

\section{Introduction}

This paper will analyse the spectrum of the Hamiltonian

$$
\hat{H}=H(\hat{x}, \hat{p})=\cos (\hat{x}-\theta)+\cos \frac{1}{2}(\hat{x}+\sqrt{3} \hat{p})+\cos \frac{1}{2}(\hat{x}-\sqrt{3} \hat{p})
$$

concentrating on results which are valid in the semiclassical limit $\hbar \rightarrow 0$. The analysis will lead to a renormalisation-group ( $R G$ ) transformation, which shows that the spectrum has a recursive structure, illustrated for $\theta=\pi / 4$ in figure 8 (see section 7 ). This hierarchical structure is a result of the classical Hamiltonian corresponding to (1.1) having centres of threefold symmetry in the $x-p$ plane. These results are related to an earlier semiclassical analysis of the spectrum of Harper's equation (Harper 1955), which is equivalent to the Hamiltonian

$$
\hat{H}=H(\hat{x}, \hat{p})=\cos \hat{x}+\cos \hat{p} .
$$

The spectrum of Harper's equation also has a recursive structure (Azbel' 1963, Hofstadter 1976), which is consequence of the fourfold symmetry of the corresponding classical Hamiltonian, and which has also been analysed by a RG transformation based on a WKB analysis (Suslov 1982, Wilkinson 1984). Although the essential ideas are the same, a more general method, with a clearer physical interpretation is described

$\dagger$ Permanent address: Department of Physics and Applied Physics, John Anderson Building, University of Strathclyde, Glasgow G4 0NG, UK. 
in this paper. The RG transformation is approximate because semiclassical methods are used to estimate certain matrix elements, but the method can be extended to give an exact RG transformation (Wilkinson 1986, 1987b). The spectrum of (1.1) has previously been examined numerically (for the case of $\theta=0$ ) by Claro and Wannier (1979), who introduced the important concept that the gaps in the spectrum can be labelled by a pair of integers, but did not consider the hierarchical properties of the spectrum.

Although the main motivation is the mathematical interest of the results, Hamiltonians periodic in $\hat{x}$ and $\hat{p}$ are also of physical interest, because they are a single-band model for Bloch electrons in a magnetic field in two dimensions (Peierls 1933, Harper, 1955, Wilkinson, 1987a), and for superconducting lattices close to the transition temperature (see e.g. Pannetier et al 1987). The rotational symmetry of the Hamiltonian in phase space corresponds to that of the lattice in real space (Wilkinson 1987a). The only cases for which a recursive spectrum occurs are when the lattice has centres of threefold or fourfold symmetry, and the models defined by (1.1) and (1.2) are universal in the sense that the fine structure of the spectrum of any Hamiltonian with these symmetries resembles that of (1.1) or (1.2), for almost all values of the $\hbar$.

The principal ideas can be summarised as follows. Because of the threefold symmetry, almost all contours of the classical Hamiltonian $H(x, p)$ are closed curves, and the energy levels are determined by the Bohr-Sommerfeld quantisation condition in the limit $h \rightarrow 0$. The Bohr-Sommerfeld quantisation condition and the form of the phase curves are discussed in section 2 . Because of the translational symmetry of the classical Hamiltonian, the Bohr-Sommerfeld quantised states are infinitely degenerate, with one state associated with each of a lattice of closed contours. The degeneracy of these states is split by tunnelling between the phase contours. Section 3 gives semiclassical formulae for the matrix elements describing this tunnelling process. In section 4 , it is shown that these matrix elements are exactly equivalent to those of an effective Hamiltonian which is of the same form as (1.1). In section 5 this result is interpreted as a renormalisation-group transformation, by which the splitting due to tunnelling of each Bohr-Sommerfeld quantised level is described by a Hamiltonian of the form (1.1), with different values of $\hbar$ and $\theta$. Section 6 describes the derivation of a simple analytical formula for the renormalisation of $\theta$. Numerical results, comparing the sub-band structure with the theoretical predictions, are described in section 7.

The method differs from that used to analyse Harper's equation (Wilkinson 1984), in that we give semiclassical estimates for the matrix elements of the Hamiltonian in a von Neumann lattice basis, with states localised in phase space, rather than in a basis in which the states are only localised in coordinate space. This canonically invariant method of calculation clarifies the role of the rotational symmetry in phase space, and greatly simplifies the calculation, which would be prohibitively difficult by the earlier method.

Because position and momentum variables play an equivalent role in our model Hamiltonian, it is important to give a canonically invariant theory for the tunnelling interaction. We give canonically invariant expressions, which can be derived in a non-rigorous way using the transfer matrix approach to WKB theory. These formulae may find applications in other problems involving systems with rotational symmetry in phase space, such as the rotational spectra of heavy, highly symmetric molecules (Harter and Patterson 1984). Our extensive numerical results provide evidence that these formulae are correct. 


\section{Phase contours and quantisation}

If the phase contours of the Hamiltonian $H(x, p)$ are closed, the energy levels are determined by the Bohr-Sommerfeld quantisation condition in the semiclassical limit $(\hbar \rightarrow 0)$. This states that the phase contour at an energy equal to the $n$th eigenvalue $E_{n}$ satisfies the area quantisation relation

$$
\oint_{H=E_{n}} p \mathrm{~d} x=2 \pi\left(n+\frac{l}{2}\right) \hbar .
$$

For a simple derivation see Landau and Lifshitz (1958). Every contour which satisfies (2.1) at a given energy corresponds to an independent solution of the Schrödinger equation: because of the invariance of the classical Hamiltonian under translations in phase space, equation (2.1) predicts that there is a discrete spectrum of infinitely degenerate levels.

It is clearly very important to understand the form of the contours of $H(x, p)$. The classical Hamiltonian has threefold rotational symmetry in phase space, and this implies that all of the phase contours are closed, apart from a separatrix at one isolated energy. Phase contours are plotted in figure $1(a)-(c)$ for three different values of $\theta$, namely $\theta=\pi / 4$, which is a typical case, and $\theta=0, \pi / 2$, which exhibit special features. Apart from an unimportant change in origin in $(x, p)$ space, the Hamiltonian changes sign upon adding $\pi$ to $\theta$ :

$$
H(x, p ; \theta+\pi)=-H\left(x^{\prime}, p^{\prime} ; \theta\right)
$$

where $x^{\prime}=x+2 \pi, p^{\prime}=p$.

Later sections of this paper will consider the splitting of the infinite degeneracy predicted by (2.1) due to tunnelling effects. To analyse these tunnelling effects it is important to characterise the possible forms for the lattice of phase trajectories at a given energy, satisfying (2.1). There are three different cases which must be considered.

(a) One contour per unit cell. This the simplest case, illustrated in figure $2(a)$. There is only one phase contour per unit cell at the energy at which the BohrSommerfeld quantisation condition is satisfied.

(b) Two contours per unit cell, non-degenerate case. This is illustrated in figure $2(b)$. There are two contours per unit cell at a given energy, with different areas. Except in cases of accidental degeneracy, only one of these contours can be made to satisfy the Bohr-Sommerfeld quantisation condition.

(c) Two contours per unit cell, degenerate case. In this case the areas of both contours satisfy the Bohr-Sommerfeld quantisation condition at the same energy, or with an energy difference comparable with the splitting due to tunnelling. For typical values of $\theta$, this only occurs for isolated values of $\hbar$, but when $\theta=0$ or $\pi$, the degeneracy is forced by the sixfold symmetry of the lattice (figure $2(c)$ ).

\section{Semiclassical theory of the tunnelling interaction}

The Bohr-Sommerfeld quantisation procedure predicts that the Hamiltonian has a discrete spectrum with infinite degeneracy. This degeneracy is split by tunnelling between the phase contours, and the discrete levels are broadened into narrow bands. The Bohr-Sommerfeld quantised states can be used as a basis set for the band, and the tunnelling interaction can be expressed in terms of a set of matrix elements coupling 
(a)
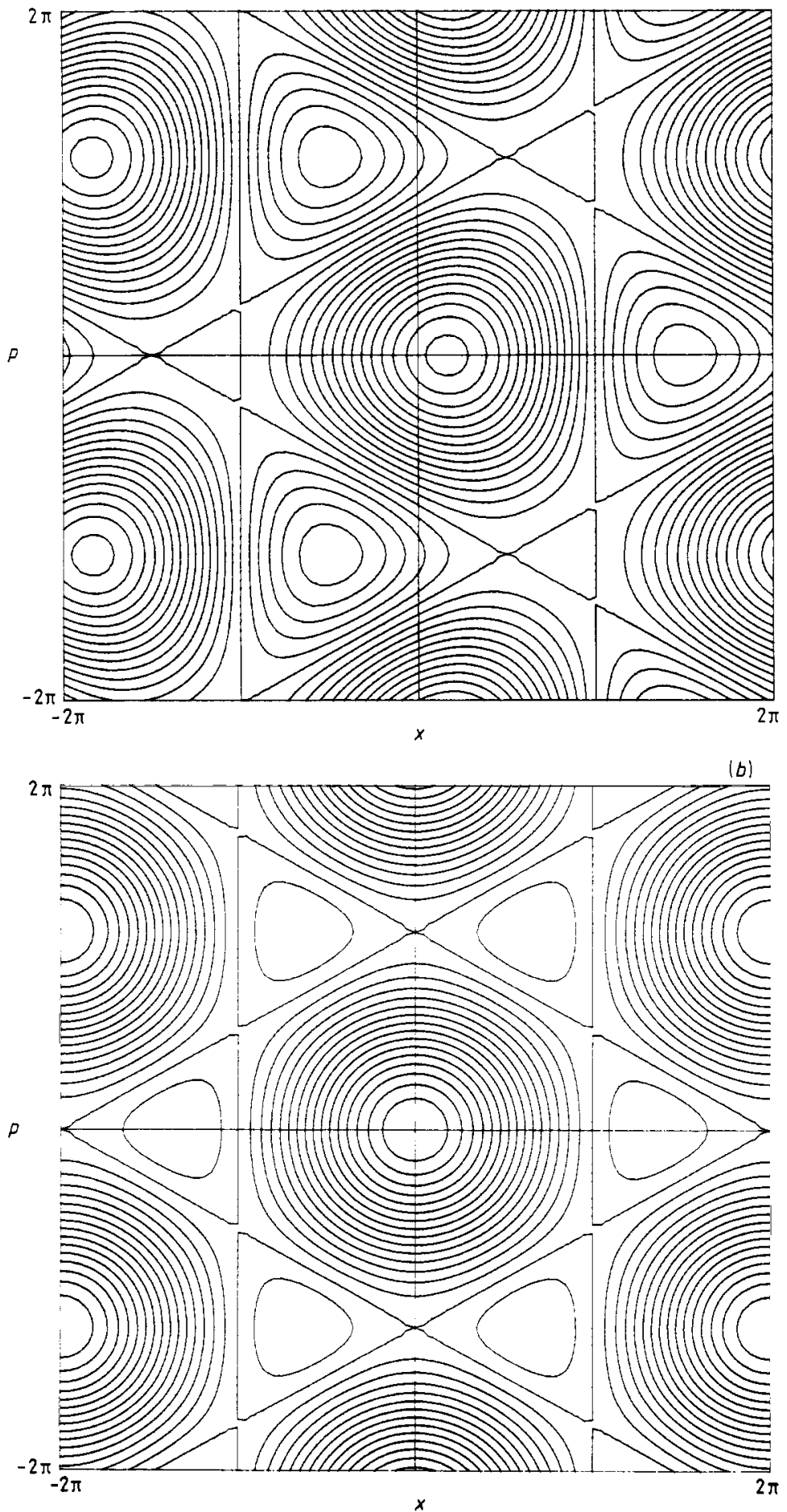

Figure 1. Contours of the classical Hamiltonian function, for various values of $\theta$ : (a) $\theta=\pi / 4$, a typical case, (b) $\theta=0$, which is special because it has sixfold symmetry, (c) $\theta=\pi / 2$, which is special because the Hamiltonian has monkey saddles. 


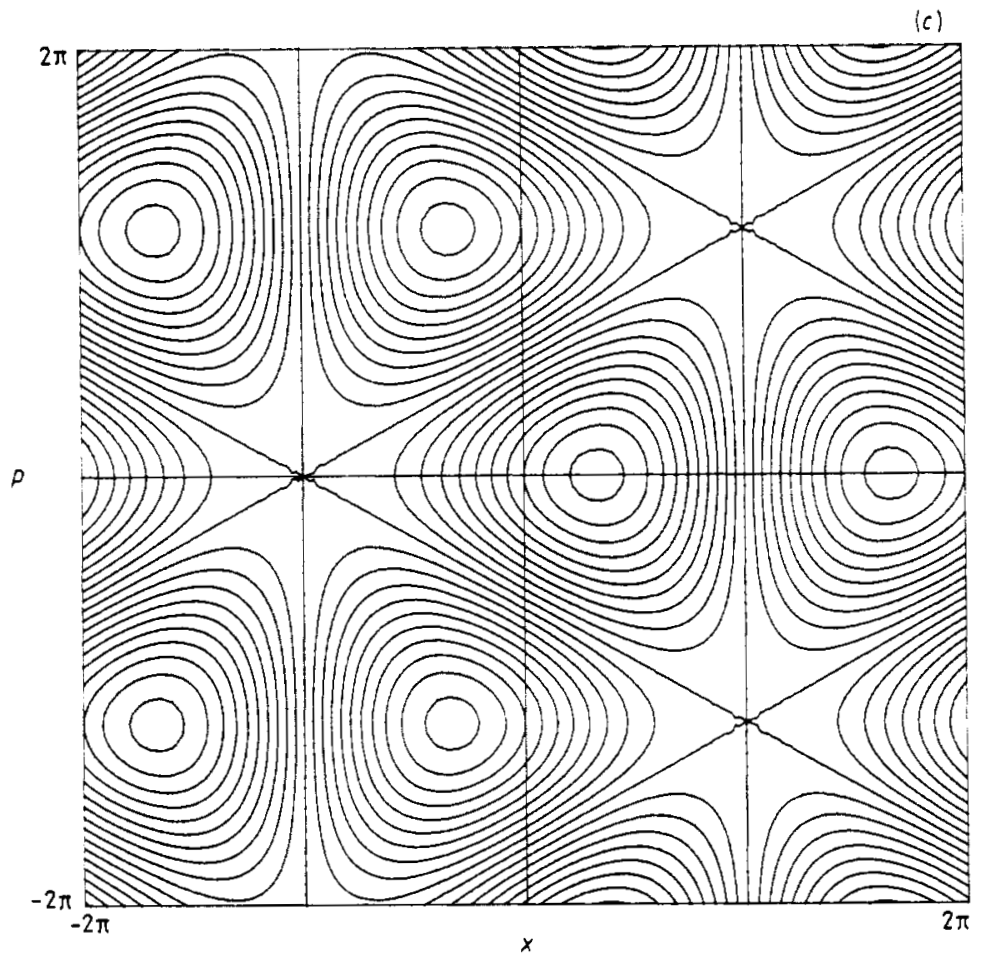

Figure 1. (continued)

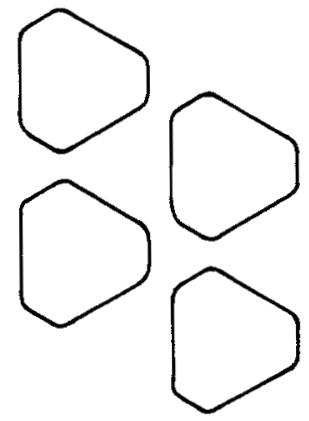

(a)

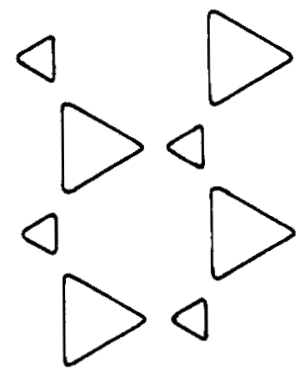

(b)

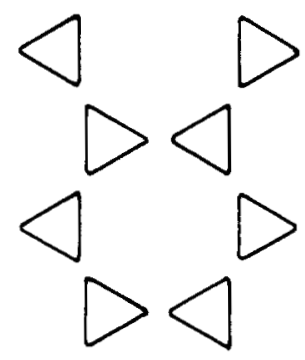

(c)

Figure 2. There are three possible forms for the contours of $H(x, p)$ at a given energy: (a) one contour per unit cell, (b) two dissimilar contours, (c) two contours per unit cell satisfying the Bohr-Sommerfeld quantisation condition.

different states in the lattice. This section will give the semiclassical theory for these matrix elements.

The matrix element coupling two Bohr-Sommerfeld quantised states has a magnitude, which is uniquely defined, and a phase which is arbitrary, because the BohrSommerfeld quantisation condition does not define the phase of the states. Although the phase of individual matrix elements is arbitrary, the sum of the phases of a sequence of matrix elements coupling a set of states cyclically does have a definite value. To 
see this, consider the states $|1\rangle,|2\rangle,|3\rangle$ shown schematically in figure 3 . Making an arbitrary choice of the phases of $|1\rangle$ and $|2\rangle$ determines the arbitrary phase of the matrix element $\langle 1|H| 2\rangle$, and an arbitrary choice of the phase of $|3\rangle$ fixes the phase of $\langle 2|H| 3\rangle$. The phase of $\langle 3|H| 1\rangle$ is then determined, so that the complex phase $\theta_{1}$ of the product of these matrix elements has a well defined value.

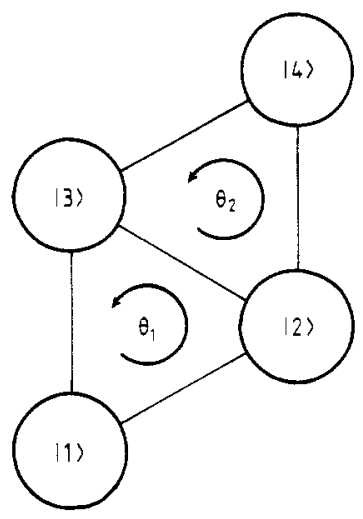

Figure 3. Because the phases of different states are arbitrary, the phase of any given matrix element is not uniquely defined. Only the product of a sequence of matrix elements joining states in a closed loop is well defined. There are two types of inequivalent irreducible loop in a simple lattice with threefold symmetry.

All the semiclassical calculations of the tunnelling matrix elements can be expressed in terms of action integrals of the form

$$
S_{C}=\oint p \mathrm{~d} q
$$

where $C$ is a closed contour of the Hamiltonian at energy $E$

$$
H(q, p)=E \text {. }
$$

The action function $S$ is invariant under canonical transformations, because $S$ is the area of the contour $C$, and canonical transformations are area preserving. Because the tunnelling matrix element can be expressed in terms of action integrals and $\hbar$, the expressions are certainly invariant under canonical transformations.

In order to calculate the tunnelling matrix elements, we must consider the analytic continuation of (3.2) into complex phase space: this equation now defines a twodimensional surface in a four-dimensional space. The contour $C$ in (3.1) is no longer unique, but the action $S$ is invariant under continuous deformations of the contour $C$, because (3.2) defines $p$ as a function of $q$ and $E$. A pair of contours $A, B$ in the real $(q, p)$ plane can usually be joined by a complex path from $A$ to $B$, every point of which lies on the complex energy surface defined by (3.2). We can form a distinct path back from $B$ to $A$ by taking the complex conjugate of the original path, which also satisfies (3.2) because $H(q, p)$ is real. The action for this loop joining contours $A$ and $B$ is clearly purely imaginary, and invariant under continuous deformations of the circuit. This action integral plays an important role in the calculation of the tunnelling matrix element.

Now we will describe the calculation of the tunnelling matrix elements for each of the patterns of phase trajectories described in section 2. In most cases the formulae used can be derived quite simply in a non-rigorous way using the transfer matrix approach to WKB theory discussed in the book by Heading (1962), and no derivation 
will be given. One formula for the semiclassical limit of a matrix element is quite difficult to derive, and this is explained in appendix $A$. The numerical methods used for analytic continuation on the complex energy surface are described in appendix $B$.

\subsection{One contour per unit cell}

In the semiclassical limit $\hbar \rightarrow 0$, the magnitude of the matrix element between states $|A\rangle$ and $|B\rangle$ is

$$
\varepsilon=\hbar\left(\omega_{A} \omega_{B}\right)^{1 / 2} \exp \left(-\left|\operatorname{Im} S_{2}\right| / \hbar\right)
$$

where $\omega_{A}$ and $\omega_{B}$ are the classical frequencies of oscillation for each phase trajectory, and $S_{2}$ is the action integral for a path on the complex energy surface joining contours $A$ and $B$ (figure $4(a)$ ). The classical frequency of vibration on a given phase trajectory is

$$
\omega_{A}=\left[\frac{\mathrm{d} S_{A}}{\mathrm{~d} E}\right]^{-1}
$$

where $S_{A}$ is the area of the phase trajectory corresponding to state $|A\rangle$ at energy $E$, and we regard $\varepsilon$ as being negative if the $\omega$ are negative. Usually there will be many different possible topologically inequivalent paths between the phase trajectories $A$ and $B$. In the limit $\hbar \rightarrow 0$, the path for which $\left|\operatorname{Im} S_{2}\right|$ is smallest makes the dominant contribution. Also, in this limit only the coupling with the largest matrix elements need be considered, since all the others are exponentially small in comparison. For all the cases we examined, the strongest coupling was between pairs of states which are geometrically nearest neighbours on the lattice.
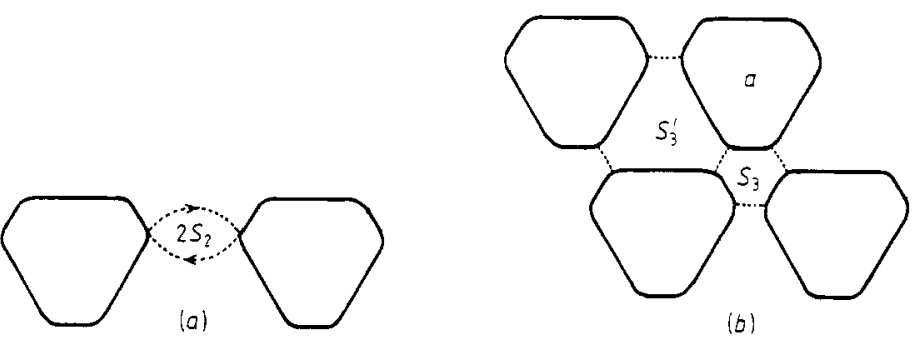

(b)

Figure 4. Paths used to describe the tunnelling theory for the coupling matrix elements. The dotted lines show the projection of paths on the complex energy surface onto the real phase plane. The paths are not unique, because the complex energy surface is twodimensional. (a) Path used to determine the action $S_{2}$, which determines the magnitude of the tunnelling matrix element. (b) Path used to determine the actions $S_{3}, S_{3}^{\prime}$ which determine the phase of the product of three matrix elements forming an irreducible cyle.

Next consider the relative phases of the matrix elements coupling the states in the lattice illustrated in figure 3. There are two distinct types of irreducible loop which can be formed by the coupling of triplets of nearest neighbouring states; the products of the matrix elements forming these loops are

$$
H_{12} H_{23} H_{31}=\varepsilon^{3} \exp \left(-\mathrm{i} \theta_{1}\right) \quad H_{24} H_{43} H_{32}=\varepsilon^{3} \exp \left(-\mathrm{i} \theta_{2}\right)
$$

where $\varepsilon$ is the magnitude of the matrix element coupling nearest neighbours in the lattice. The phases $\theta_{1}$ and $\theta_{2}$ are both calculated in the same way: we find a path on 
the complex energy surface joining all three states in a loop (figure $4(b)$ ). If the action integral for this path is $S_{3}$, the phase $\theta$ is given by

$$
\theta=\left(\operatorname{Re}\left(S_{3}\right) / \hbar+\pi / 2\right) \bmod 2 \pi \text {. }
$$

\subsection{Two contours per unit cell, non-degenerate case}

In this case, only one of the contours in each unit cell satisfies the Bohr-Sommerfeld quantisation condition. The other contour is still important, because although it does not support a quantum state at this energy, the tunnelling path uses this contour. The magnitude of the coupling matrix element between states on sublattice $A$ is given by

$$
\varepsilon=\frac{\hbar \omega_{A} t^{2}}{2 \sin (\gamma / 2)}
$$

where

$$
\gamma=\frac{S_{B}}{\hbar}+\pi \quad t=\exp \left(-\left|\operatorname{Im} S_{2}\right| / \hbar\right)
$$

$S_{B}$ is the action integral of the contour on sublattice $B$, and $S_{2}$ is the action integral joining phase contours of the $A$ and $B$ states. Note that this expression diverges when the contours of the $B$ sublattice satisfy the Bohr-Sommerfeld quantisation condition, because this condition can be written in the form $\sin (\gamma / 2)=0$. A derivation of (3.7) is given in appendix $A$.

The relative phases of the matrix elements are characterised by two phase angles as in the case of a lattice with one contour per unit cell. In this case the phase $\theta$ for the smaller loop (containing only one contour of type $B$ ) is

$$
\theta=-\frac{\gamma}{2}+\pi\left[\frac{\gamma}{2 \pi}\right]
$$

where $[X]$ denotes the integer part of $X$.

\subsection{Two contours per unit cell, degenerate case}

In this case all the contours support quantum states at the energy concerned, and the magnitude $\varepsilon$ of the tunnelling matrix element is given by (3.3). The smallest irreducible circuits of coupled states contain six states, and there is only one type of loop for which the product of the matrix elements is

$$
H_{12} H_{23} H_{34} H_{45} H_{56} H_{61}=\varepsilon^{6} \exp (-\mathrm{i} \theta) \text {. }
$$

The phase $\theta$ is obtained from a path connecting all six of these contours on the complex energy surface: if $S_{6}$ is the action integral of this closed path, then

$$
\theta=\left(\operatorname{Re} S_{6} / \hbar\right) \bmod 2 \pi
$$

and simple geometrical considerations show that

$$
\operatorname{Re} S_{6}=A-a_{1}-a_{2}
$$

where $A$ is the area of the unit cell, and $a_{1}, a_{2}$ are the areas of the two contours at energy $E$. Because the areas of these contours satisfy the Bohr-Sommerfeld quantisation condition, then

$$
S_{6}=A \bmod 2 \pi \hbar
$$


which implies that we can take

$$
\theta=A / \hbar \text {. }
$$

\section{An effective Hamiltonian for the tunnelling interaction}

The results of the previous section showed how to calculate the matrix elements describing the tunnelling process in the limit $\hbar \rightarrow 0$. In this limit, only tunnelling between nearest-neighbouring states (for which $|\operatorname{Im} S|$ is smallest) need be considered; matrix elements coupling other pairs of states are negligible. The phases of the matrix elements are not determined uniquely; only the sum of the phases for a sequence of matrix elements forming a closed loop is physically meaningful. This section will show how it is possible to write down an effective Hamiltonian for which the matrix elements are equivalent to those of the tunnelling interaction, and which is closely related to the original Hamiltonian (1.1). There are two cases, which must be considered separately.

(a) Simple lattice (figure $5(a)$ ). The interaction is described by four parameters: $E_{0}$, the unperturbed energy of the states; $\varepsilon$, the magnitude of the tunnelling matrix element, and the phases $\theta_{1}, \theta_{2}$ of the matrix elements for the two inequivalent loops. The parameters $E_{0}$ and $\varepsilon$ only determine a shift of origin and scale of the spectrum, and we can set $E_{0}=0, \varepsilon=1$, without loss of generality. (If $\varepsilon$ is negative, we must add $\pi$ to $\theta_{1}$ and $\theta_{2}$ to take account of this; see (3.5).)
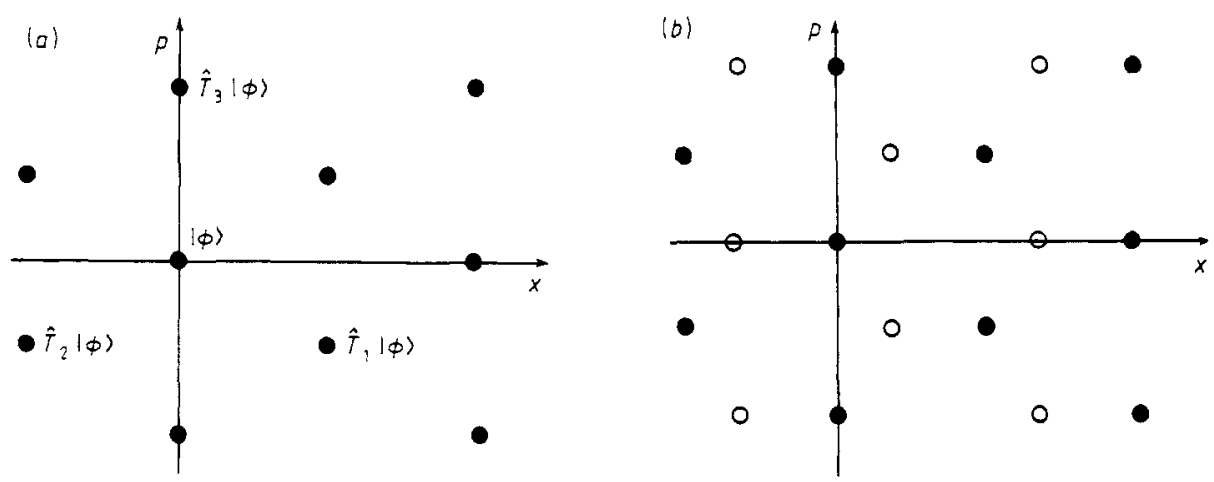

Figure 5. Lattices used to define the effective Hamiltonian for the tunnelling interaction: (a) simple lattice, (b) dual lattice.

(b) Dual lattice (figure 5(b)). The interaction is again described by four parameters: the unperturbed energies $E_{A}, E_{B} \cdot \mathrm{f}$ the different types of states, the magnitude of the matrix elements $\varepsilon$, and a phase $\theta$ for the single type of loop. Without loss of generality, we can reduce this to two parameters: we set the origin and scale of the spectrum by taking $E_{0}=\left(E_{A}+E_{B}\right) / 2=0$, and $\varepsilon=1$. The remaining two parameters are $\theta$ and $\Delta=\left(E_{A}-E_{B}\right) / \varepsilon$.

\subsection{Simple lattice case}

The strategy is to define a von Neumann lattice of states based on the simple triangular lattice illustrated in figure 5(a), and to consider the matrix elements of a Hamiltonian 
of the form (1.1) in this basis. The matrix elements are equivalent to those of the tunnelling interaction derived above, for a suitable choice of $\theta$ and $\hbar$.

First consider the generation of a von Neumann lattice of states. The operator

$$
\hat{T}(X, P)=\exp [\mathrm{i}(P \hat{x}-X \hat{p}) / \hbar]
$$

has the properties of a translation operator in phase space; it shifts the Wigner function of the state on which it acts by the vector $R=(X, P)$ in phase space. The lattice illustrated in figure $5(a)$ is based on three fundamental translations $T_{1}, T_{2}$, and $T_{3}$, represented by the operators

$\hat{T}_{1}=\hat{T}(2 \pi,-2 \pi / \sqrt{ } 3) \quad \hat{T}_{2}=\hat{T}(-2 \pi,-2 \pi / \sqrt{ } 3) \quad \hat{T}_{3}=\hat{T}(0,4 \pi / \sqrt{ } 3)$.

We define a von Neumann lattice of states $|n, m\rangle$ by translation of a generating state $|\phi\rangle$, which has its Wigner function localised around an arbitrary origin in phase space:

$$
|n, m\rangle=\hat{T}_{2}^{m} \hat{T}_{1}^{n}|\phi\rangle
$$

and assume that the generating state is chosen so that these states are orthogonal

$$
\left\langle n^{\prime}, m^{\prime} \mid n, m\right\rangle=\delta_{n n^{\prime}} \delta_{m m} .
$$

The translation operators (4.1) do not commute; using the Baker-Hausdorf relation we see that if $\boldsymbol{R}_{1}, \boldsymbol{R}_{2}, \boldsymbol{R}_{3}$ are three vectors in the phase plane which form a closed loop

$$
\boldsymbol{R}_{1}+\boldsymbol{R}_{2}+\boldsymbol{R}_{3}=\mathbf{0}
$$

then

$$
\hat{T}\left(\boldsymbol{R}_{3}\right) \hat{T}\left(\boldsymbol{R}_{2}\right) \hat{T}\left(\boldsymbol{R}_{1}\right)=\exp (\mathrm{i} A / \hbar)
$$

where $A$ is the area of the triangle formed by the three vectors, with the convention that $A$ is positive if the boundary of the triangle is traversed anticlockwise.

Having defined the von Neumann lattice, we now introduce a Hamiltonian which is the sum of the three elementary lattice translations and their inverses

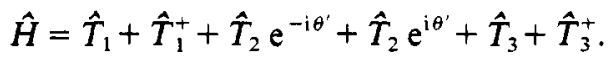

Note that this Hamiltonian connects each coherent state in the von Neumann lattice to all of its nearest neighbours. Let us evaluate the product of the matrix elements of (4.7) for the two cyclic sequences of states illustrated in figure 3 . Using the results above

$$
\begin{aligned}
H_{12} & =\langle 1|\hat{H}| 2\rangle=\left\langle 1\left|\hat{H} \hat{T}_{2}^{+}\right| 1\right\rangle=\exp \left(-\mathrm{i} \theta^{\prime}\right) \\
H_{23} & =\langle 2|\hat{H}| 3\rangle=\left\langle 1\left|\hat{T}_{2} \hat{H} \hat{T}_{3}\right| 1\right\rangle=\left\langle 1\left|\hat{T}_{2} \hat{T}_{1} \hat{T}_{3}\right| 1\right\rangle \\
& =\exp (-\mathrm{i} A / 2 \hbar) \\
H_{31} & =\langle 3|\hat{H}| 1\rangle=\left\langle 1\left|\hat{T}_{3}^{+} \hat{H}\right| 1\right\rangle=1
\end{aligned}
$$

where $A$ is the area of the unit cell, equal to $8 \pi^{2} / \sqrt{ } 3$. The product of the matrix elements for the first cycle is therefore

$$
H_{12} H_{23} H_{31}=\exp \left[-\mathrm{i}\left(A / 2 \hbar+\theta^{\prime}\right)\right] \text {. }
$$

A similar calculation shows that the product for the second cycle is

$$
H_{43} H_{32} H_{24}=\exp \left[-\mathrm{i}\left(A / 2 \hbar-\theta^{\prime}\right)\right] \text {. }
$$


It is clear that the matrix elements of the Hamiltonian (4.7) are equivalent to the matrix elements calculated semiclassically in section 3 if

$$
\begin{aligned}
& \theta_{1}=\left(A / 2 \hbar+\theta^{\prime}\right) \bmod 2 \pi \\
& \theta_{2}=\left(A / 2 \hbar-\theta^{\prime}\right) \bmod 2 \pi
\end{aligned}
$$

and that (4.7) can therefore be used as an effective Hamiltonian for describing the splitting of the Bohr-Sommerfeld quantised states.

Using (4.1), we see that the effective Hamiltonian can be written in the form

$$
\hat{H}=2 \cos \left(\hat{x}^{\prime}-\theta^{\prime}\right)+2 \cos \frac{1}{2}\left(\hat{x}^{\prime}+\sqrt{ } 3 \hat{p}^{\prime}\right)+2 \cos \frac{1}{2}\left(\hat{x}^{\prime}-\sqrt{ } 3 \hat{p}^{\prime}\right)
$$

where $x^{\prime}, p^{\prime}$ are scaled coordinates and momenta

$$
x^{\prime}=\frac{4 \pi^{2}}{\sqrt{3} \hbar} x \quad p^{\prime}=\frac{4 \pi^{2}}{\sqrt{3} \hbar} p
$$

which satisfy the commutation relation

$$
\left[\hat{x}^{\prime}, \hat{p}^{\prime}\right]=\mathrm{i} \frac{16 \pi^{2}}{3 \hbar}=\mathrm{i} \hbar^{\prime} .
$$

The splitting of the band by tunnelling effects is therefore described by an effective Hamiltonian (4.12) which is identical to the original one, apart from having a different phase parameter $\theta^{\prime}$ and a different effective Planck constant $\hbar^{\prime}$.

\subsection{Dual lattice case}

The dual lattice is illustrated in figure $5(b)$. There are two interpenetrating simple lattices of the type illustrated in figure $5(a)$. The states localised on the $A$ and $B$ sublattices have site energies $\Delta / 2$ and $-\Delta / 2$ respectively, and the matrix elements coupling nearest neighbours are taken to have unit magnitude. In section 3 , we saw that the product of the matrix elements coupling a cycle of six states has a complex phase which is equal to the area of a unit cell divided by $\hbar$, modulo $2 \pi$ (compare with 3.14)):

$$
H_{12} H_{23} H_{34} H_{45} H_{56} H_{61}=\exp (-\mathrm{i} \theta) \quad \theta=A / \hbar-2 \pi n .
$$

In the case of the dual lattice our strategy will be to calculate the matrix elements of the square of the effective Hamiltonian. We will find that this is equal to the effective Hamiltonian for a simple lattice with a particular value of $\theta^{\prime}$, plus a constant term. The matrix elements of the square of the Hamiltonian are

$$
\left\langle a\left|\hat{H}^{2}\right| b\right\rangle=\sum_{i}\langle a|\hat{H}| i\rangle\langle i|\hat{H}| b\rangle
$$

where the sum runs over all possible intermediate states $|i\rangle$. Three cases must be considered. giving

(a) Diagonal matrix elements, $|a\rangle=|b\rangle$. Here four terms contribute to the sum,

$$
\left\langle a\left|H^{2}\right| a\right\rangle=3+\Delta^{2} / 4 \text {. }
$$

(b) States $|a\rangle$ and $|b\rangle$ are nearest neighbours. Here two equal and opposite terms contribute to the matrix element, which is therefore zero. 


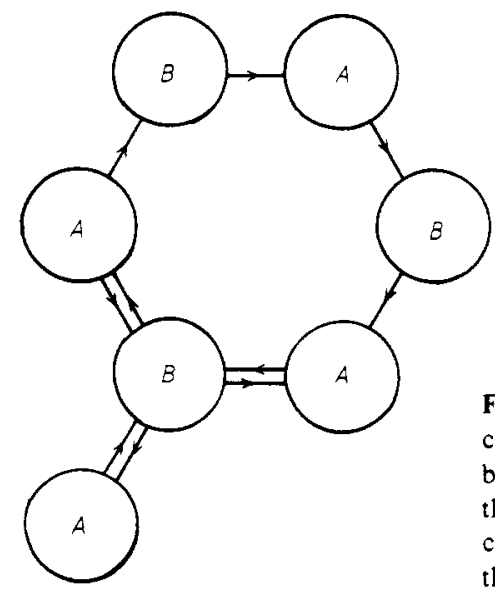

Figure 6. Illustrating the two types of irreducible cycles which occur when the tunnelling paths between states on the $A$ sublattice use contours of the $B$ sublattice. One type of cycle uses one $B$ contour to connect three $A$ states, and the other uses three $B$ contours.

(c) States $|a\rangle$ and $|b\rangle$ are next-nearest neighbours. Here only one term contributes, and the magnitude of the matrix element is unity. The product of three matrix elements of $H^{2}$ coupling a cycle of states is given by (4.15) for one type of irreducible circuit and unity for the other type, in which the three states on the $A$ sublattice are coupled through only one contour of the $B$ sublattice (see figure 6). For all other pairs of states the matrix elements of $H^{2}$ are zero.

Note that the next-nearest neighbours of sites on the dual lattice are nearest neighbours on the simple lattice, and that the matrix element coupling nearest neighbours is zero. This shows that the square of the Hamiltonian describing the tunnelling splitting is equal to that given by (4.7) or (4.12), plus a constant $3+\Delta^{2} / 4$, given by (4.17). The effective Hamiltonian is therefore

$\hat{H}=\left[\left(3+\Delta^{2} / 4\right)+2 \cos \left(\hat{x}^{\prime}-\theta^{\prime}\right)+2 \cos \frac{1}{2}\left(\hat{x}^{\prime}+\sqrt{3} \hat{p}^{\prime}\right)+2 \cos \frac{1}{2}\left(\hat{x}^{\prime}-\sqrt{3} \hat{p}^{\prime}\right)\right]^{1 / 2}$

with

$$
\theta^{\prime}=\frac{A}{2 \hbar}
$$

where the commutator of $\hat{x}^{\prime}$ and $\hat{p}^{\prime}$ is given by (4.14). Both branches of the square root are required, one for each of the two sublattices.

\section{The renormalisation-group transformation}

In this section we review the important results from sections 3 and 4 and show how they can be interpreted as a renormalisation-group transformation acting on the parameters $\theta$ and $\hbar$. Section 3 gave semiclassical expressions for the matrix elements of the tunnelling interaction, and section 4 showed that these matrix elements are the same as those of an effective Hamiltonian coupling the states of a von Neumann lattice. The effective Hamiltonian (4.12) is of the same form as the original Hamiltonian (1.1), which suggests that a renormalisation-group transformation exists for describing the fine structure of the spectrum.

We cannot, however, conclude immediately that we have constructed a renormalisation-group transformation. The difficulty is that the von Neumann lattice basis was 
an undercomplete set (with less than one state per Planck constant area). The remaining part of the argument, which resolves this difficulty, was discussed in an earlier paper (Wilkinson 1986). This paper considered the matrix elements of a Hamiltonian $\hat{H}$, periodic in $\hat{x}$ and $\hat{p}$, in an undercomplete von Neumann lattice basis, $|n, m, \nu\rangle$. The von Neumann lattice basis was transformed into a new basis, $|n, \delta, \nu\rangle$ (essentially, this is a Fourier transformation over the $m$ label, with $\delta$ regarded as a continuous variable with a finite range), and it was shown that in this new basis the Hamiltonian becomes a difference operator with periodic coefficients. An effective Hamiltonian (analogous to that considered above) was written down, and it was shown that taking matrix elements with respect to a suitable complete basis leads to the same difference equation. This establishes that the spectrum of the sub-band is indeed that of the effective Hamiltonian.

The results of section 4 therefore imply that the splitting of the Bohr-Sommerfeld quantised level has the same spectrum as a renormalised Hamiltonian, with different values of $\hbar$ and $\theta$, which can be calculated using the semiclassical results from section 3. The remainder of this section gives explicit formulae for the renormalised parameters $\theta^{\prime}, \hbar^{\prime}$ : the discussion is divided into three parts, depending on the form of the lattice of phase trajectories.

\subsection{One contour per unit cell}

The phase relationship of the matrix elements coupling the Bohr-Sommerfeld quantised states is described by two phase angles, which give the complex phase of the product of the matrix elements for the two types of irreducible loops in the lattice. The semiclassical theory for these phase angles is described in section 3 ; the phase angles $\theta_{1}, \theta_{2}$ are given by

$$
\theta_{1}=\frac{S_{3}}{\hbar} \pm \frac{\pi}{2} \quad \theta_{2}=\frac{S_{3}^{\prime}}{\hbar} \pm \frac{\pi}{2}
$$

where we take the positive sign if the contours are below the separatrix, and vice versa. The action integrals satisfy

$$
S_{3}+S_{3}^{\prime}=A-a
$$

where $A=8 \pi^{2} / \sqrt{ } 3$ is the area of the unit cell, and $a$ is the area of the Bohr-quantised contour (see figure $4(b)$ ).

The coupling matrix elements were shown in section 4 to be equivalent to those of an effective Hamiltonian, which is a sum of translation operators acting on a von Neumann lattice corresponding to the Bohr-Sommerfeld quantised states. The corresponding phases of products of matrix elements are

$$
\theta_{1}=\frac{A}{2 \hbar}+\theta^{\prime} \quad \theta_{2}=\frac{A}{2 \hbar}-\theta^{\prime}
$$

where $\theta^{\prime}$ is a parameter of the effective Hamiltonian. Note that (5.1) and (5.2), together with the Bohr-Sommerfeld condition satisfied by $a$, imply that $\theta_{1}+\theta_{2}$ is equal to $A / \hbar \bmod 2 \pi$, as is required for consistency with (5.3). Comparing (5.1) and (5.3) we find an expression for $\theta^{\prime}$ :

$$
\theta^{\prime}=\frac{A}{2 \hbar}-\frac{S_{3}}{\hbar} \mp \frac{\pi}{2}
$$


By a suitable scaling of the phase space coordinates $x$ and $p$, the effective Hamiltonian can be written in the same form as the original Hamiltonian:

$$
H^{\prime}=\cos \left(\hat{x}-\theta^{\prime}\right)+\cos \frac{1}{2}(\hat{x}+\sqrt{ } 3 \hat{p})+\cos \frac{1}{2}(\hat{x}-\sqrt{ } 3 \hat{p}) .
$$

The effective value of $\hbar$ is

$$
\hbar^{\prime}=\frac{16 \pi^{2}}{3 \hbar}
$$

and the area of the transformed unit cell is $A^{\prime}=\sqrt{3} \hbar^{\prime 2} / 2$.

It is useful to introduce a dimensionless measure of the phase associated with the area of a unit cell, $\beta$, defined by

$$
\frac{A}{\hbar}=\frac{A^{\prime}}{\hbar^{\prime}}=\frac{2 \pi}{\beta} \text {. }
$$

In terms of $\beta$, the transformation of the effective Planck constant (5.6) can be expressed in the simple form

$$
\beta^{\prime}=\frac{1}{\beta}
$$

where from (5.7) $\beta=\sqrt{ } 3 \hbar / 4 \pi$ and $\beta^{\prime}=\sqrt{ } 3 \hbar^{\prime} / 4 \pi$.

The splitting of the degeneracy of the Bohr-Sommerfeld quantised states is determined by a new effective Hamiltonian of the same form as the original one, but with new values of $\theta$ and $\beta$ (or $\hbar$ ). Equations (5.4) and (5.8) define a renormalisation-group transformation mapping the parameters $\beta$ and $\theta$. The conditions for the validity of these expressions are those required for the validity of the semiclassical approximations used in sections 2 and 3 , i.e. that $\beta$ or $\hbar$ is small and the energy is not close to that of the separatrix. Equation (5.8) appears to contradict the possibility of iterating this transformation, because the semiclassical condition requires that $\beta^{\prime}$ should also be small. Equations (5.4) and (5.8) are not a unique choice of the renormalisation mapping, however: if we subtract an integer $N$ from $\beta^{\prime}$, this causes a change $N \pi$ in the product of phases of the matrix elements in each irreducible cycle, which must be compensated by adding $N \pi$ to $\theta_{1}$ and $\theta_{2}$

$$
\beta^{\prime}=\frac{1}{\beta}-N \quad \theta^{\prime}=\frac{A}{2 \hbar}-\frac{S_{3}}{\hbar} \mp \frac{\pi}{2}+N \pi .
$$

We choose $N$ to be the closest integer to $1 / \beta$, so that we obtain the smallest value of $\beta^{\prime}$. This transformation of $\beta$ is closely related to its continued fraction expansion (Wilkinson 1984). If $\beta^{\prime} \ll 1$, we can perform further iterations of this semiclassical renormalisation-group transformation.

\subsection{Two contours per unit cell, non-degenerate case}

This case is essentially the same as the case of one contour per unit cell, except that the phase of the product of matrix elements is given by (3.9) instead of (3.6). The expression for the renormalisation of $\theta$ must be altered accordingly:

$$
\theta^{\prime}=\pi \beta^{\prime}+\frac{\gamma}{2}-\pi\left[\frac{\gamma}{2 \pi}\right]
$$

where $\beta^{\prime}$ is the renormalised value of $\beta$, given by (5.9), and $\gamma=\left(S_{B} / \hbar\right)+\pi$. 


\subsection{Two contours per unit cell, degenerate case}

If there are two contours per unit cell supporting Bohr-Sommerfeld quantised states, the renormalisation-group transformation can be described in similar terms to that of the simple lattice, and the results are simpler. The renormalised effective Hamiltonian is given by

$\hat{H}^{\prime}=\left[\left(3+\Delta^{2}\right)+2 \cos \left(\hat{x}-\theta^{\prime}\right)+2 \cos \frac{1}{2}\left(\hat{x}+\sqrt{3} \hat{p}^{\prime}\right)+2 \cos \frac{1}{2}\left(\hat{x}-\sqrt{3} \hat{p}^{\prime}\right)\right]^{1 / 2}$

for which the spectrum is just a folded version of that of (5.5), since if $E$ belongs to the spectrum of $\hat{H}$ then $f(E)$ belongs to the spectrum of $f(\hat{H})$. The renormalisation equations for $\beta$ are simply

$$
\beta^{\prime}=\frac{1}{\beta}-N \quad \theta^{\prime}=\pi \beta^{\prime}
$$

where $N$ is the closest integer to $1 / \beta$.

If $\theta=0$, the lattice has sixfold symmetry, and at those energies where there are two contours per unit cell these contours are automatically degenerate. It is interesting to note that this sixfold symmetry is broken by the RG transformation, as (5.12) shows that the renormalised Hamiltonian does not have $\theta^{\prime}=0$, and therefore has threefold symmetry.

\section{Renormalisation of the phase parameter}

In this section we discuss an analytical result for the renormalisation of the phase parameter $\theta$. The result depends on a special feature of the model Hamiltonian (1.1), and we do not expect that an analytical form for $\theta^{\prime}$ could be found for a general Hamiltonian with threefold symmetry. It is, however, important to analyse this special case because of the universality of our model: the effective Hamiltonian describing the splitting of a Bohr-Sommerfeld quantised sub-band is always of the form of (1.1) whatever threefold symmetric model we start from, provided $\beta$ is small so that our semiclasssical theory applies. Furthermore, the iteration of the mapping (5.9) describing the renormalisation of $\beta$ produces arbitrarily small values of $\beta^{\prime}$ for a typical initial value (Wilkinson 1984).

To calculate $\theta^{\prime}$ we must calculate the action integral $S_{3}$ or $S_{3}^{\prime}$. The crucial observation leading to an analytical expression for $S_{3}$ is that as the energy changes both $S_{3}$ and $S_{3}^{\prime}$ change by the same amount. A proof of this result will be given later, but first we continue to give a formula for $S_{3}$. The sum of $S_{3}$ and $S_{3}^{\prime}$ is given by (5.3), so that these actions are given by

$$
S_{3}=\alpha-a / 2 \quad S_{3}^{\prime}=\alpha^{\prime}-a / 2
$$

where $a$ is the area of the phase trajectory and $\alpha, \alpha^{\prime}$ are constants. These constants can be fixed by noting that at the energy of the separatrix the segments of the path defining $S_{3}$ on the complex energy surface vanish, and $S_{3}$ is equal to the area of one of the real triangular phase contours. The areas of these triangular contours are $(\pi-2 \theta)^{2} / \sqrt{ } 3$ and $(\pi+2 \theta)^{2} / \sqrt{ } 3$ and the area of the unit cell is $8 \pi^{2} / \sqrt{ } 3$. At the energy of the separatrix, therefore

$$
a=A-\frac{(\pi-2 \theta)^{2}}{\sqrt{ } 3}-\frac{(\pi+2 \theta)^{2}}{\sqrt{ } 3} \quad S_{3}=\frac{(\pi-2 \theta)^{2}}{\sqrt{ } 3}
$$


so that, by comparison with (6.1),

$$
S_{3}=\frac{4 \pi(\pi-\theta)}{\sqrt{ } 3}-\frac{a}{2} .
$$

This equation can be used together with (5.9) to give an analytical formula for $\theta^{\prime}$ which avoids the numerical evaluation of the action integral.

We must now consider the proof of the assertion contained in (6.1), that changing the energy (and hence $a$ ) causes equal changes in $S_{3}$ and $S_{3}^{\prime}$. Let us consider a path on the complex energy surface joining the three contours illustrated in figure $7 ; S_{3}$ is the real part of the action integral for this path. We join contours $A$ and $B$ by a path which holds $\operatorname{Im}(p)=0$, allowing $x$ to become complex, and the segments from contours $A$ to $C$ and $B$ to $C$ hold $\operatorname{Im}(x)=0$, allowing $p$ to be complex. The real part of the action integral, $S_{3}$, is just the area of the curve formed by the projection of this path onto the real $x-p$ plane. The Hamiltonian (1.1) has the special feature that the momentum can be expressed as a function of the position analytically:

$$
H(x, p)=\cos (x-\theta)+2 \cos (x / 2) \cos (\sqrt{ } 3 p / 2)
$$

so that

$$
p=\frac{2}{\sqrt{ } 3} \cos ^{-1}\left(\frac{E-\cos (x-\theta)}{2 \cos (x / 2)}\right) .
$$

If we fix $\operatorname{Im}(x)=0$, we see that outside the classically allowed regions, $\operatorname{Re}(p)=2 \pi n / \sqrt{3}$, where $n$ is an odd or even integer depending on the sign of the argument of the $\cos ^{-1}$ function. The real projection of the path defining $S_{3}$ therefore has the form shown by the bold line in figure 7. The shaded area in figure 7 is, by symmetry, equal to one third of $S_{3}$, and it is geometrically obvious that this area plus one sixth of the area of the phase contour is equal to a constant as the energy is varied, i.e. $S_{3} / 3+a / 6=$ constant in agreement with (6.1).

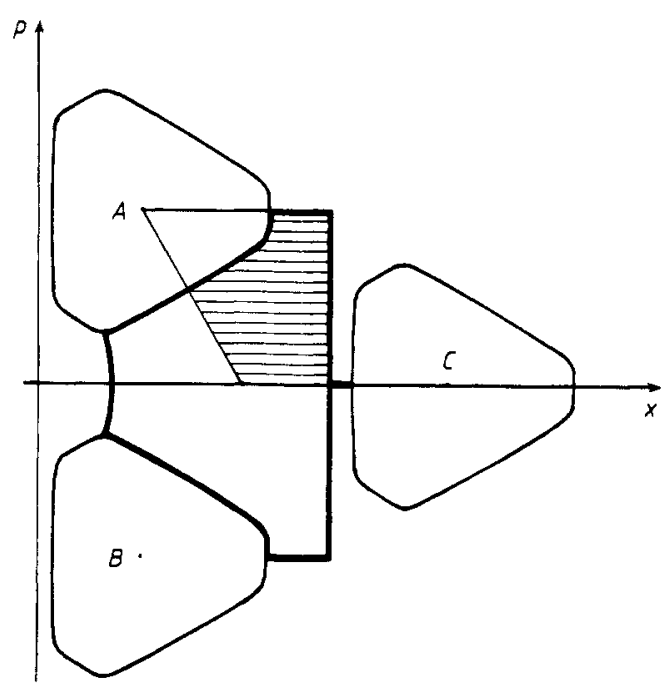

Figure 7. Illustrating the proof of a formula for the action integral $S_{3}$ for the model Hamiltonian used. See section 6 for an explanation. 
The discontinuity of $\operatorname{Re}(p)$ at the point where $\cos (x / 2)=0$ is associated with a logarithmic divergence of $\operatorname{Im}(p)$. A path on the complex energy surface which does not diverge to infinity can be obtained by adding a very small imaginary part to $x$; this prevents $\operatorname{Im}(p)$ from diverging but has a negligible effect on the behaviour of $\operatorname{Re}(p)$. This completes our discussion of the derivation of (6.3).

Equation (6.3) can be used to derive an explicit formula for $\theta^{\prime}$ when there is one contour per unit cell, because $a$ must be a half-integer multiple of $h$ in order to satisfy Bohr-Sommerfeld quantisation:

$$
\begin{aligned}
\theta^{\prime} & =\frac{A}{2 \hbar}-\frac{S_{3}}{\hbar} \mp \frac{\pi}{2}+N \pi \\
& =\frac{\theta A}{2 \pi \hbar}+\left(n+\frac{1}{2} \mp \frac{1}{2}\right) \pi+N \pi \\
& =\theta\left(\beta^{\prime}+N\right)+\left(N+n+\frac{1}{2} \mp \frac{1}{2}\right) \pi
\end{aligned}
$$

(the negative sign applies if the contours are below the separatrix).

\section{Numerical results}

Numerical experiments were performed to compare the semiclassical theory with the exact quantum mechanical spectrum. The exact evaluation of the spectrum was carried out by quantising the Hamiltonian in a coordinate representation, in which case the Schrödinger equation becomes a difference equation with periodic coefficients (Wilkinson 1984, 1986). Bloch's theorem is applicable when $\beta$ is a rational number, $N / M$, where the integers $N, M$ are coprime; in this case the spectrum consists of exactly $M$ bands. When $\beta$ is irrational the spectrum is a Cantor set. All the numerical examples refer to rational values of $\beta$.

Figure 8 illustrates the complexity of the spectrum; this is a plot of the energy bands for all rational $\beta=N / M$, with $M \leqslant 40$. The sub-bands corresponding to BohrSommerfeld quantised energy levels can be seen clearly, and it is clear that the width of these bands vanishes very quickly as $\hbar \rightarrow 0$. It is also possible to see that the sub-bands have a structure which resembles that of the whole spectrum, but which is not exactly self-similar.

The detailed numerical results are divided into three sections, corresponding to the three cases distinguished in section 3.

\subsection{One contour per unit cell}

Table 1 shows some semiclassical estimates of the parameters of the effective Hamiltonian describing the splitting of various sub-bands. The interpretation of the columns is as follows: $\theta, \beta$, and $\hbar=4 \pi \beta / \sqrt{ } 3$ are parameters of the original Hamiltonian, $n$ is the Bohr-Sommerfeld quantisation number, and $\omega=(\partial S / \partial E)^{-1}$ is the classical frequency for the sub-band concerned (positive for bands below the separatrix, negative for those above). The energy predicted by Bohr-Sommerfeld quantisation is $E_{n}, S_{2}$ and $S_{3}$ are the action integrals which characterise the tunnelling effect, and $\varepsilon_{\text {pred }}$ is the theoretically predicted magnitude of the tunnelling matrix element, and $\varepsilon_{\text {obs }}$ is the value deduced from the numerically computed width of the sub-band. The parameters of the renormalised Hamiltonian are $\theta^{\prime}, \beta^{\prime}$ and $\hbar^{\prime}=4 \pi \beta / \sqrt{ } 3$. We computed the actions 


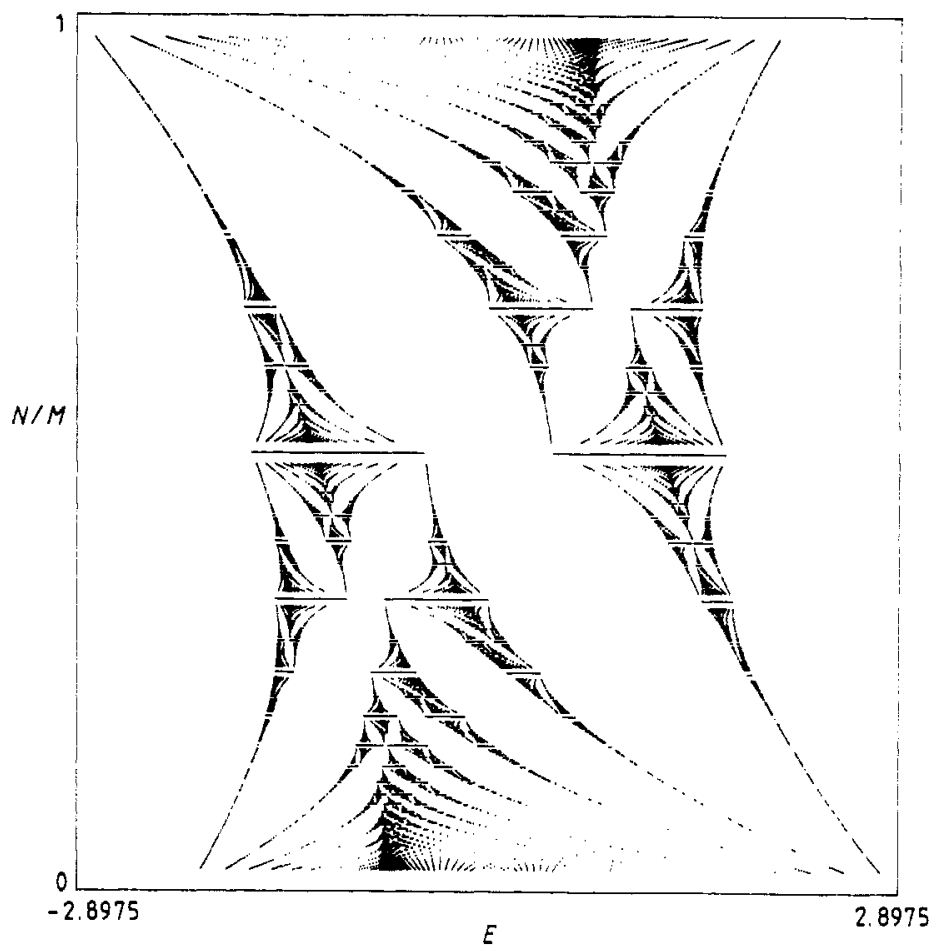

Figure 8. Plot illustrating the complex structure of the spectrum, for a typical value of $\theta$, $\theta=\pi / 4$. The spectrum can only be computed when $\beta$ is a rational number, $N / M$, when it consists of $M$ bands. These bands are plotted as horizontal lines for every rational $\beta$ with $M \leqslant 40$.

Table 1. Parameters for numerically computed examples in figure 9.

\begin{tabular}{lccc}
\hline Example & $a$ & $b$ & $c$ \\
\hline$\theta / \pi$ & 0 & 0.5 & 0.25 \\
$\beta$ & $19 / 100$ & $19 / 100$ & $15 / 151$ \\
$\hbar$ & 1.378 & 1.378 & 0.721 \\
$n$ & 0 & 0 & 2 \\
$E_{n}$ & 2.052 & 1.786 & 0.834 \\
$\omega$ & -0.200 & -0.169 & -0.139 \\
$S_{2}$ & 17.233 & 14.707 & 10.336 \\
$S_{3}$ & 43.420 & 9.231 & 11.438 \\
$\varepsilon_{\text {pred }}$ & $5.32 \mathrm{E}-4$ & $1.12 \mathrm{E}-3$ & $7.69 \mathrm{E}-5$ \\
$\varepsilon_{\text {obs }}$ & $5.22 \mathrm{E}-4$ & $1.06 \mathrm{E}-3$ & $8.31 \mathrm{E}-5$ \\
$\theta^{\prime} / \pi$ & 1 & 0.368 & 0.515 \\
$\beta^{\prime}$ & $5 / 19$ & $5 / 19$ & $1 / 15$ \\
\hline
\end{tabular}

$S_{3}$ numerically, using the method described in appendix $B$, and found good agreement with the analytical formula, (6.3).

The spectra of the renormalised Hamiltonians specified in table 1 are compared with those of the sub-bands they model in figure $9(a)-(c)$ : the spectrum of the original Hamiltonian is plotted above, with an expanded view of one sub-band (centre). The 


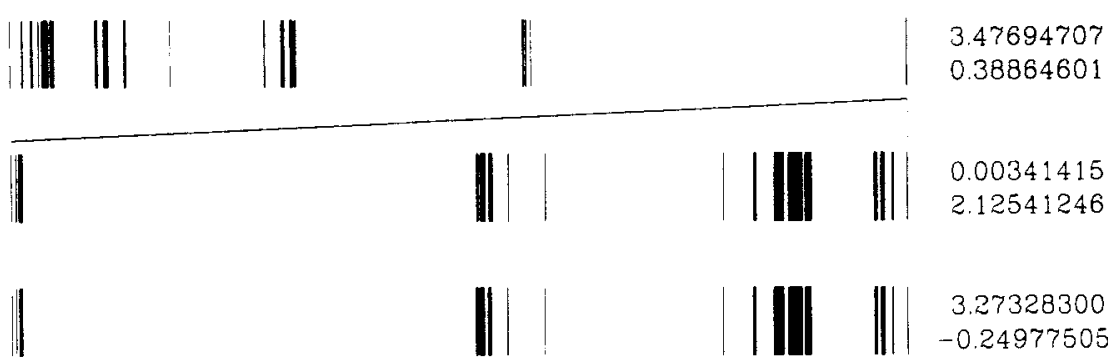

(a)

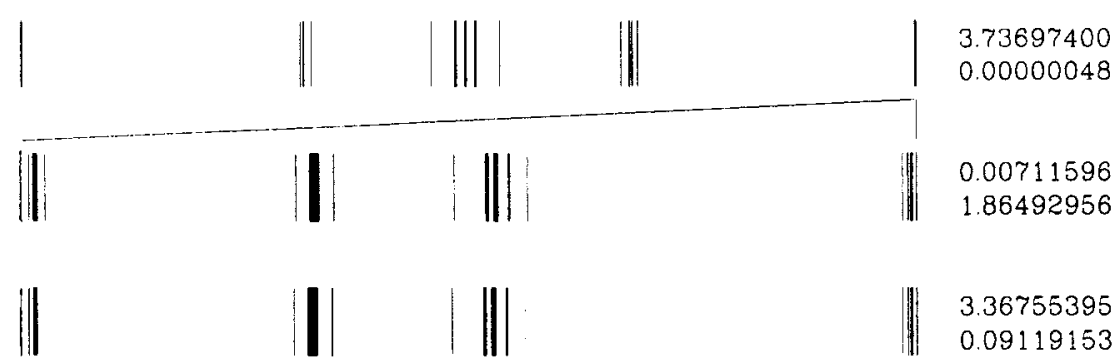

(b)

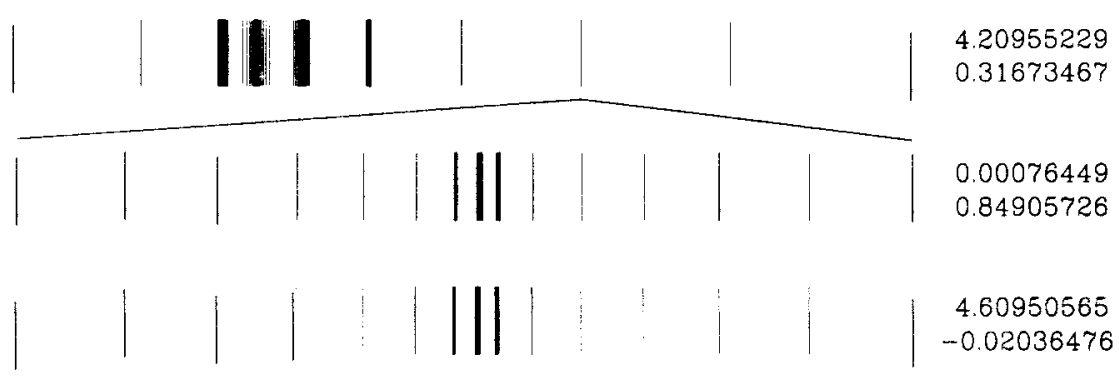

(6)

Figure 9. Comparison of the spectrum of the renormalised Hamiltonian (below) with the sub-band (centre) of the original Hamiltonian which it is intended to model (above); the numbers to the right are the widths and the positions of the centre of each spectrum. The other parameters are listed in table 1. All of these examples are of sub-bands for which there is one phase contour per unit cell.

spectrum of the renormalised Hamiltonian with the same spectrum as this sub-band is shown below. All the spectra are scaled and shifted so that they have the same range: the numbers to the right are the widths and the positions of the centres of the unscaled spectra.

\subsection{Two contours per unit cell, non-degenerate case}

Results for this case are shown in table 2. The interpretation of most of the columns is the same, except that $S_{3}$ is replaced by $S_{B}$, the area of the second phase trajectory, which does not satisfy the Bohr-Sommerfeld condition. The comparisons of spectra are shown in figure $10(a)-(c)$; the interpretation is the same as for figure 9. 
Table 2. Parameters for numerically computed examples in figure 10 .

\begin{tabular}{llll}
\hline Example & $a$ & $b$ & $c$ \\
\hline$\theta / \pi$ & 0.03 & 0.03 & 0.11 \\
$\beta$ & $5 / 106$ & $7 / 150$ & $5 / 151$ \\
$\hbar$ & 0.342 & 0.339 & 0.240 \\
$n$ & 0 & 1 & 0 \\
$E_{n}$ & -1.302 & -1.229 & -1.123 \\
$\omega$ & 0.102 & 0.094 & 0.087 \\
$S_{2}$ & 1.249 & 0.915 & 0.770 \\
$S_{B}$ & 2.445 & 1.820 & 5.771 \\
$\varepsilon_{\text {pred }}$ & $1.33 \mathrm{E}-5$ & $8.05 \mathrm{E}-5$ & $2.00 \mathrm{E}-5$ \\
$\varepsilon_{\text {obs }}$ & $1.41 \mathrm{E}-5$ & $7.31 \mathrm{E}-5$ & $1.93 \mathrm{E}-5$ \\
$\theta^{\prime} / \pi$ & 0.837 & 0.784 & 0.523 \\
$\beta^{\prime}$ & $1 / 5$ & $3 / 7$ & $1 / 5$ \\
\hline
\end{tabular}

\subsection{Two contours per unit cell, degenerate case}

This case occurs naturally in the case of a Hamiltonian with sixfold symmetry (i.e. $\theta=0$ ), and can also occur due to accidental degeneracy when there is threefold symmetry. We show two examples where the degeneracy is symmetry induced $(\theta=0)$, and a third where this degeneracy is broken by changing $\theta$ slightly. In this case we can estimate $\Delta$, the offset of two nearly degenerate bands, using the Bohr-Sommerfeld quantisation rule:

$$
\Delta=2 \omega\left[\frac{\partial S}{\partial \theta}\right]_{E} \theta / \varepsilon
$$

where $\partial S / \partial \theta$ is the rate of change of the area of a phase contour with $\theta$. The parameters calculated semiclassically are given in table 3 ; this has the same format as table 1 . The comparison of spectra is shown in figure $11(a)-(c)$.

\section{Acknowledgments}

Many of the ideas were developed during a visit to the Centre de Physique Theorique, CNRS, Luminy, Marseille, and we are greateful to Professor $\mathrm{J}$ Bellissard for his hospitality there, and to the Nuffield Foundation for grants for computer equipment. This work was supported in part by the US National Science Foundation.

\section{Appendix A}

In this appendix we discuss the calculation of the effective matrix element for tunnelling between three identical Bohr-Sommerfeld quantised levels, in the case where the tunnelling path uses a fourth contour, which does not satisfy the Bohr-Sommerfeld condition at the energy concerned (see figure 12).

The amplitudes of the case letters in figure 12 . We consider the amplitudes $a, b, a^{\prime}, b^{\prime}$, which are related to each other because of tunnelling between the phase contours, and calculate the small 


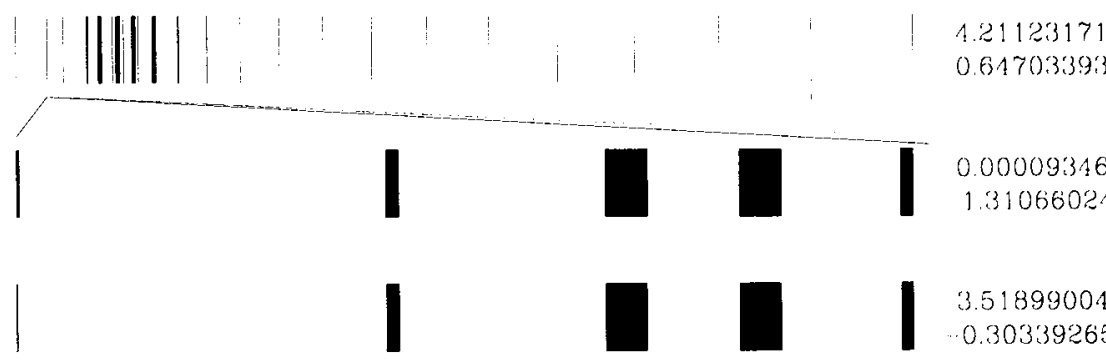

(a)

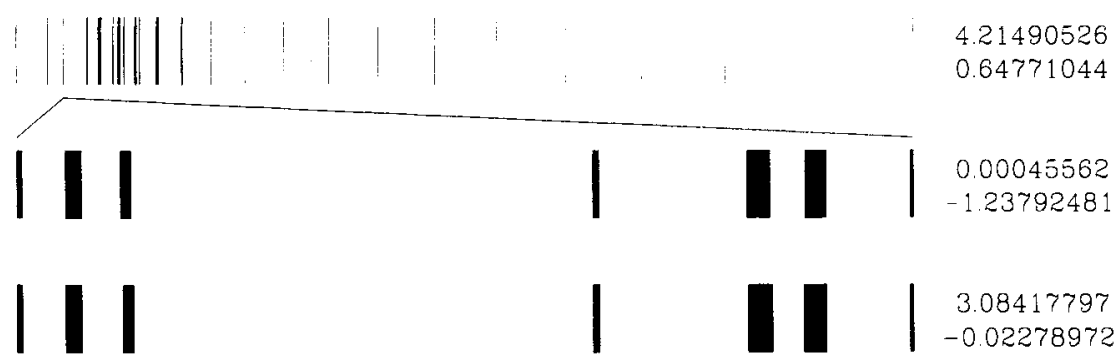

(b)

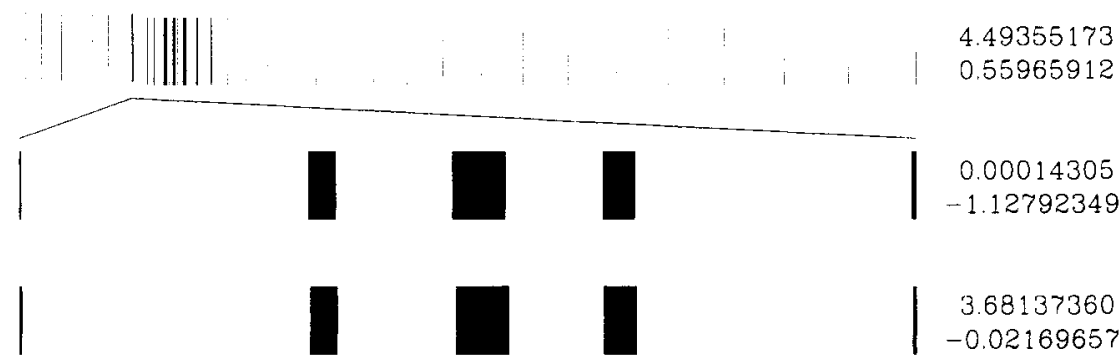

(c)

Figure 10. Similar to figure 9: the sub-bands are at an energy where there are two non-degenerate phase contours per unit cell. The other parameters are listed in table 2.

amplitudes $a, b$ assuming that $a^{\prime}, b^{\prime}$ are of order unity. These amplitudes are related by a transfer matrix (Heading 1962):

$$
\left(\begin{array}{l}
a \\
b
\end{array}\right)=\frac{\mathrm{i}}{t}\left(\begin{array}{cc}
1 & -r \\
r & 1
\end{array}\right)\left(\begin{array}{l}
a^{\prime} \\
b^{\prime}
\end{array}\right)
$$

where $t$ is the transmission amplitude for the tunnelling process, and $r$ is the magnitude of the reflection coefficient

$$
t=\exp \left(-\left|\operatorname{Im} S_{2}\right| / \hbar\right) \quad t^{2}+r^{2}=1 .
$$

The amplitude $b^{\prime}$ has the same magnitude as $a^{\prime}$ :

$$
b^{\prime}=-a^{\prime} \exp (\mathrm{i} \phi) \quad \phi=S_{A} / \hbar=2 \pi\left(n+\frac{1}{2}\right)+\delta
$$

where $S_{A}$ is the area of the uppermost phase contour, and $\delta$ is a correction to the 
Table 3. Parameters for numerically computed examples in figure 11.

\begin{tabular}{llll}
\hline Example & $a$ & $b$ & $c$ \\
\hline$\theta / \pi$ & 0 & 0.0001 & 0 \\
$\beta$ & $7 / 150$ & $7 / 150$ & $8 / 193$ \\
$\hbar$ & 0.339 & 0.339 & 0.301 \\
$n$ & 0 & 0 & 0 \\
$E_{n}$ & -1.379 & -1.379 & -1.392 \\
$\omega$ & 0.109 & 0.109 & 0.110 \\
$S_{2}$ & 1.578 & 1.578 & 1.644 \\
$\Delta$ & 0 & 1.418 & 0 \\
$\varepsilon_{\text {pred }}$ & $3.49 \mathrm{E}-4$ & $3.49 \mathrm{E}-4$ & $1.40 \mathrm{E}-4$ \\
$\varepsilon_{\text {obs }}$ & $3.51 \mathrm{E}-4$ & $3.55 \mathrm{E}-4$ & $1.44 \mathrm{E}-4$ \\
$\theta^{\prime} / \pi$ & 0.429 & 0.429 & 0.125 \\
$\beta^{\prime}$ & $3 / 7$ & $3 / 7$ & $1 / 8$ \\
\hline
\end{tabular}

Bohr-Sommerfeld quantisation condition, which embodies the tunnelling effect. Combining the above expressions, and assuming that $\delta$ and $t$ are small, we find:

$$
\left(\begin{array}{l}
a \\
b
\end{array}\right)=\left(\begin{array}{l}
\delta / t+\mathrm{i} t / 2 \\
\delta / t-\mathrm{i} t / 2
\end{array}\right)
$$

so that

$$
b=\exp (\mathrm{i} \chi) a \quad \tan (\chi / 2)=t / 2 \delta^{2} .
$$

Each of the three tunnelling events causes a phase shift of $\chi$ of the wavefunction on the central phase contour. The overall change in phase of this wavefunction as it is traced around the central phase contour should be equal to an integer multiple of $2 \pi$ :

$$
2 \pi n=3 \chi+\gamma \quad \gamma=S_{B} / \hbar+\pi
$$

where $S_{B}$ is the area of the central phase trajectory and the additional phase change of $\pi$ is because this trajectory has two turning points. The phase shift $\delta$ is therefore

$$
\delta=\frac{t^{2}}{2} \cot (\gamma / 6+n \pi / 3)
$$

which takes three distinct values. The change in the energy of the eigenstate due to the phase change $\delta$ is

$$
\Delta E=\hbar \omega_{A} \delta \quad \omega_{A}=\left[\frac{\partial S_{A}}{\partial E}\right]^{-1}
$$

so that the threefold degeneracy is split as follows:

$$
E_{n}=E_{0}+\frac{\hbar \omega_{A} t^{2}}{2} \cot (n \pi / 3+\gamma / 6)
$$

where $E_{0}$ is the energy predicted by Bohr-Sommerfeld quantisation.

Now we relate the splitting given by (A9) to a model in which we assume that each of the three states is coupled to its two neighbours by a matrix element of magnitude $\varepsilon$, with the product of the matrix elements having phase $\theta$ :

$$
H_{12} H_{23} H_{31}=\varepsilon^{3} \exp (-\mathrm{i} \theta) \text {. }
$$




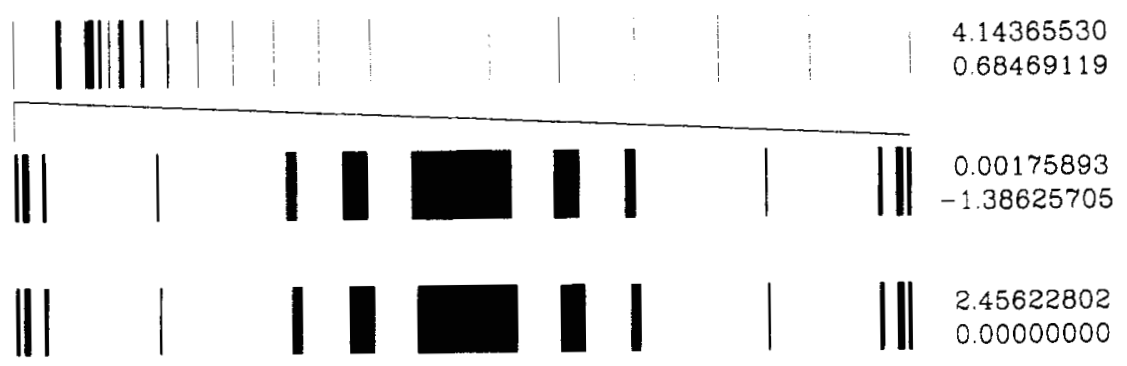

(a)
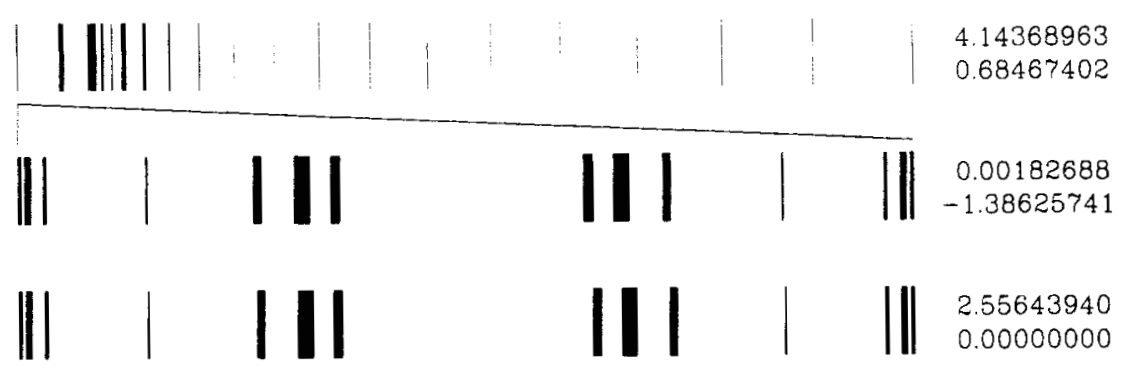

(b)

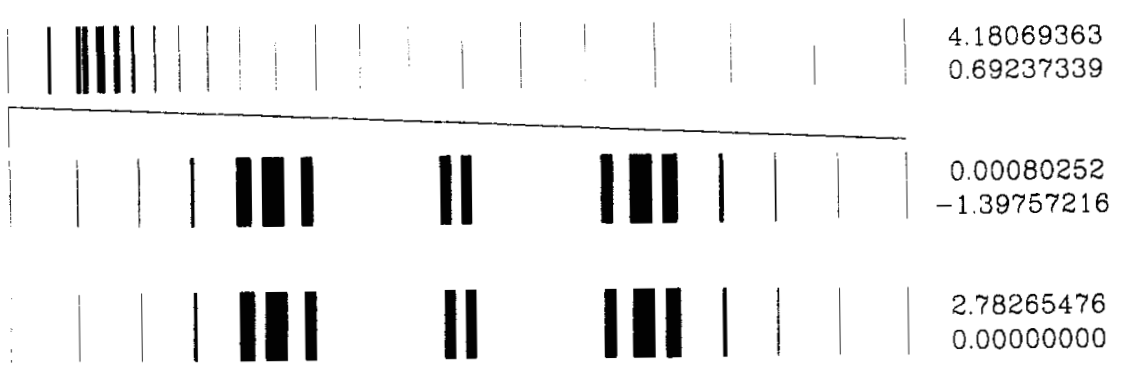

(c)

Figure 11. Similar to figure 9: in these examples there are two degenerate phase contours per unit cell. Table 3 lists the other parameters.

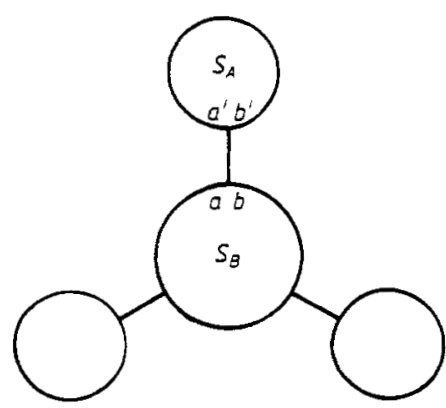

Figure 12. Phase contours for three Bohr quantised states, for the case described in appendix $A$ where the tunnelling path is via a fourth, non-resonant contour. The lower case letters stand for the amplitude of the WKB wavelets at various points. 
The energy levels of this model are

$$
E_{n}=E_{0}^{\prime}+2 \varepsilon \cos [2 \pi n / 3+\theta / 3]
$$

where $E_{0}^{\prime}$ is the constant diagonal matrix element. We choose parameters $E_{0}^{\prime} \varepsilon, \theta$ of the model so the energy levels agree with (A9). We find the ratio $\left(E_{1}-E_{2}\right) /\left(E_{2}-E_{3}\right)$ is in agreement if we set

$$
\theta=-\frac{\gamma}{2}+\pi\left[\frac{\gamma}{2 \pi}\right]
$$

where $[X]$ is the integer part of $X$, and that $E_{1}-E_{2}$ is in agreement if

$$
\varepsilon=\frac{\hbar \omega_{A} t^{2}}{2 \sin (\gamma / 2)} .
$$

Clearly $E_{0}^{\prime}$ is approximately equal to $E_{0}$. The calculation of the difference between these quantities using the formalism above is meaningless; there are corrections to the Bohr-Sommerfeld condition with a power-law dependence on $\hbar$, starting at order $\hbar^{3}$, which are much larger than the exponentially small corrections due to tunnelling. The power-law corrections depend only on the properties of one real phase trajectory, and therefore make no contribution to the splitting of degeneracies, which is therefore determined by tunnelling effects.

\section{Appendix B}

In this appendix we describe the method used to evaluate the complex-valued action integrals which are required for the calculation of the tunnelling matrix elements. This material is included because the complex energy surface has a complicated geometry, and some obvious numerical methods will not work.

The problem is to calculate an integral of the form

$$
S=\int p(q ; E) \mathrm{d} q
$$

where the function $p(q ; E)$ is defined implicitly by

$$
H(q, p)=E
$$

and the beginning and end points of the integration are points on two different real contours of the Hamiltonian. An obvious method for evaluating this integral would be to define the values of $\operatorname{Re} q$ and $\operatorname{Re} p$ along a path between the two contours, and to adjust the values of $\operatorname{Im} q$ and $\operatorname{Im} p$ at each point along the path so that $(\mathrm{A} 2)$ is always satisfied (this can be achieved using a Newton-Raphson method). This method will fail if there are no solutions of (A2) for given values of $\operatorname{Re} q, \operatorname{Re} p$ at some point along the path. Due to the geometry of the complex energy surface, it is always found that the imaginary parts of $q$ and $p$ do not return to zero at the end of the path, i.e. we end up on a complex branch of the energy surface, rather than the real contour we were aiming for. The reason for this surprising observation is understood, but would require a lengthy discussion.

Instead of prescribing $\operatorname{Re} q$ and $\operatorname{Re} p$ along the tunnelling path, we make a canonical transformation, in the form of a rotation of the phase plane, so that the projections onto the $q$ axis of the two contours being joined do not overlap. We then calculate 


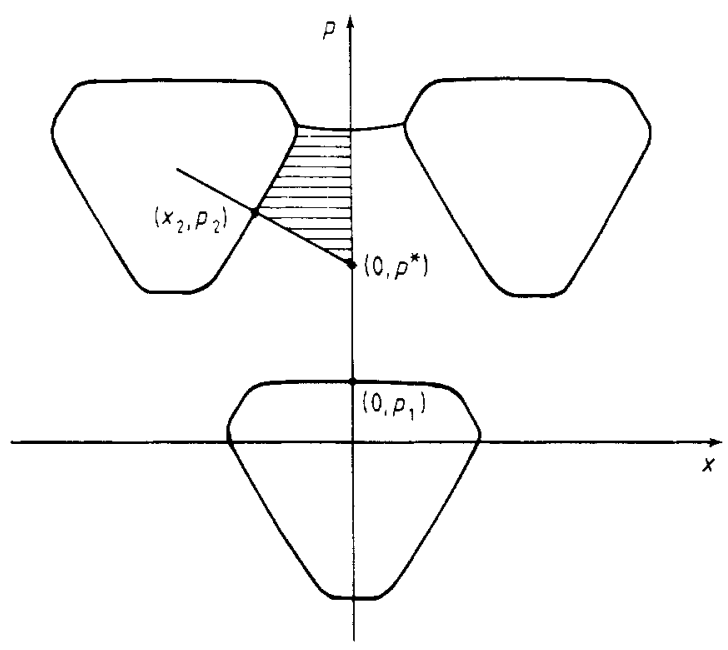

Figure 13. Illustrates the method described in appendix B which was used to calculate $S_{2}$ and $S_{3}$ numerically.

the integral with $p$ complex but with $\operatorname{Im} q=0$. This always brings us back onto a real branch of the energy surface.

As an example, we describe in detail how we calculate the tunnelling action $S_{2}$ and the action $S_{3}$ determining the relative phases of the matrix elements, for the case of a simple lattice of states. First we rotate the Hamiltonian by $\pi / 2$ in the phase plane by the transformation $(x, p) \rightarrow\left(x^{\prime}, p^{\prime}\right)=(p,-x)$. The rotated Hamiltonian has mirror symmetry about the line $x=0$. We locate a point $\left(0, p^{*}\right)$ of threefold symmetry along this line (this can be done analytically), and we find the value $p_{1}$ of the momentum closest to this point of symmetry such that the point $x=0, p=p_{1}$ lies on the phase contour at energy $E$ (see figure 13 ). We rotate the point $\left(0, p_{1}\right)$ about the centre of symmetry to find another point $\left(x_{2}, p_{2}\right)$ on a phase contour at energy $E$. We then evaluate the action integral

$$
\Sigma=\int_{0}^{x_{2}} \mathrm{~d} x p(x ; E) .
$$

Both $S_{2}$ and $S_{3}$ can be obtained from $\Sigma$; clearly

$$
S_{2}=2 \operatorname{Im} \Sigma
$$

and it is obvious from figure 13 on geometrical grounds that

$$
2 \operatorname{Re} \Sigma=S_{3} / 3+x_{2}\left(p^{*}+p_{2}\right) \text {. }
$$

\section{References}

Azbel' M Ya 1964 Zh. Eksp. Teor. Fiz. 46929 (Engl. transl. Sov. Phys.-JETP 19 634-45)

Claro F and Wannier G H 1979 Phys. Rev. B 19 6068-74

Harper P G 1955 Proc. Phys. Soc. A 68 874-92

Harter W G and Patterson C W 1984 J. Chem. Phys. 80 4241-61

Heading J 1962 An Introduction to Phase Integral Methods (London: Methuen) 
Hofstadter D R 1976 Phys. Rev. B $142239-49$

Landau L D and Lifshitz E M 1958 Quantum Mechanics (Oxford: Pergamon)

Pannetier B, Chaussy J and Rammal R 1987 Japan. J. Appl. Phys. 26 suppl 26-3 1994-8

Peierls R 1933 Z. Phys. 80 763-91

Suslov I M 1982 Z. Eksp. Teor. Fiz. 83 1079-88 (Engl. transl. Sov. Phys.-JETP 56 612-17)

Wilkinson M 1984 Proc. Roy. Soc. A 391 305-50

1986 Proc. Roy. Soc. A 402 135-66

- 1987a J. Phys. A: Math. Gen. 20 1761-71

- 1987b J. Phys. A: Math. Gen. 20 4337-54 